Ann. Biol. anim. Bioch. Biophys., I968, 8 (3), 349-36o.

\title{
ACTION DU SYSTÈME NERVEUX SUR L'EXTENSION PIGMENTAIRE CONSÉCUTIVE A L'AUTOGREFFE DE PEAU CHEZ LE COBAYE
}

\author{
C. VOULOT \\ Laboratoire de Biologie, \\ Institut national des Sciences appliquées, 69 - Villeurbanne
}

SOMMAIRE

L'influence du système nerveux cutané sur le phénomène d'extension pigmentaire consécutif à l'autogreffe de peau pigmentée en territoire blanc, a été étudiée chez le Cobaye.

- Si l'on réalise une énervation de la région qui reçoit le greffon pigmenté, l'extension pigmentaire est plus importante que là où l'innervation a été laissée en place.

- Les faits expérimentaux peuvent être expliqués en envisageant une action inhibitrice de l'innervation cutanée sur le phénomène d'extension pigmentaire.

- Lors de l'autogreffe, l'excision du lit porte-greffe entraîne une énervation locale. Nous avons vérifié que si l'on augmente la taille du lit porte-greffe, la surface atteinte par l'extension pigmentaire est plus importante.

\section{INTRODUCTION}

Le phénomène d'extension pigmentaire chez le Cobaye, connu depuis longtemps, ne fut étudié en détail qu'à partir de I948 par BIL,INGHAN et MEDAWAR.

Rappelons-en les grandes lignes; si de la peau noire d'un cobaye tricolore est greffée sur un territoire blanc du même animal, on voit la tache pigmentée correspondant à la greffe, s'étendre progressivement. Nous avons montré (VouLoT, I965), que cette extension se déroule d'abord à vitesse constante (jusqu'au $3^{\mathrm{e}}$ mois environ) puis ralentit et finit par s'arrêter au bout d'un temps plus ou moins long (vers le $6^{\mathrm{e}}$ mois).

Après que diverses hypothèses aient été émises, il semble qu'il faille considérer l'extension pigmentaire comme l'effet d'une migration des mélanocytes (BII,LINGHAM et Silvers, I964). Mais l'étude des différents mécanismes physiologiques impliqués reste cependant à faire. 
Il est plausible d'envisager dans un premier temps une action du système nerveux cutané ; en effet les auteurs qui décrivirent l'extension pigmentaire, notèrent que la vitesse de cette extension est plus grande, après action dans la zone intéressée d'agents irritants : incisions, application d'acétone ou de térébenthine, etc.

FABIAN en I953 après avoir entouré d'une incision continue, la zone où legreffon est implanté, constate une extension pigmentaire plus importante que pour les greffes témoins. L'auteur interprète cette différence comme le résultat d'une énervation consécutive à l'incision.

Nous avons repris l'expérimentation de FABIAN en utilisant outre sa technique, une énervation plus spécifique et mieux définie, et en appliquant les méthodes quantitatives précédemment mises au point (VOULOT, I965). Cette étude nous a conduit à émettre l'hypothèse d'un freinage del'extension pigmentaire sous l'influence de l'innervation superficielle. Il est d'autre part évident que l'excision du lit porte-greffe, préalable à l'implantation du greffon, lèse cette innervation superficielle. En conséquence, notre hypothèse impliquait l'existence d'une relation entre les limites atteintes par l'extension pigmentaire, et la surface du lit porte-greffe initial. Nous avons cherché à vérifier cette relation, ce qui constitue la detuxième partie de ce travail.

\section{MATÉRIEL, E'T TECHNIQUES}

\section{Techniques de greffe}

Les interventions chirurgicales sont toutes pratiquées sous anesthésie au Nembutal, l'animal étant placé sur une table chauffante.

La greffe de peau est réalisée comme précédemment (VouloT, 1965).

Cependant lorsque le lit porte-greffe doit être de diamètre très supérieur au greffon ; la technique diffère légèrement. La surface excisée, non recouverte par le greffon noir est alors uniformément complétée par des greffons de peau blanche obtenus en taillant le lit porte-greffe lui-même (voir fig. I A).

Le greffon noir est toujours disposé sur le bord du lit porte-greffe.

\section{Technique d'énervation}

\section{a) Anatomie de l'innervation cutanée du Cobaye.}

Nous n'avons considéré que la région du flanc, limitée à l'avant par la $\mathbf{I}^{\text {re }}$ côte asternale, et à l'arrière par la pointe du coxal.

Le tégument des Mammifères est innervé par les branches des nerfs rachidiens et d'une manière générale :

- la branche dorsale innerve les zones dorso-latérales,

- le rameau perforant de la branche ventrale, innerve les régions latéro-ventrales.

Au niveau des flancs, on a donc 2 séries de nerfs cutanés émergeant suivant 2 alignements antéropostérieurs.

Nous avons vérifié cette disposition chez le Cobaye. Les branches dorsales des $9^{\mathrm{e}}$ aux $\mathrm{I} 3^{\mathrm{e}}$ paires thoraciques, émergent un peu au-dessous du trapèze dorsal. Les rameaux perforants des branches ventrales des $8^{\mathrm{e}}$ aux $13^{\mathrm{e}}$ paires thoraciques, émergent au niveau du bord postérieur des insertions costales du grand oblique de l'abdomen. Dans le prolongement de l'alignement des branches dorsales thoraciques, on note l'émergence des branches dorsales des premières paires lombaires de nerfs rachidiens. De la même façon, on trouve dans le prolongement de l'alignement des rameaux perforants thoraciques, les rameaux des premières paires lombaires. 
b) Exploration électrophysiologique des dermatomes correspondant aux nerfs superficiels.

Nous avons utilisé un poste fixe d'électrophysiologie (Racia), comprenant :

- un dispositif de pré- et post-amplification,

- un oscilloscope à double trace, présentant une sensibilité maximale de $10 \mu \mathrm{V}$ par $\mathrm{cm}$,

- un ensemble de haut-parleurs incorporés.

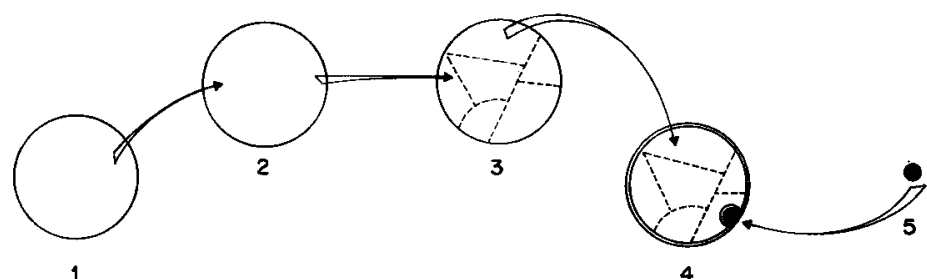

A

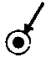

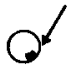

b

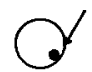

$c$

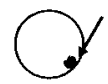

d

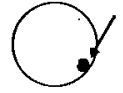

e

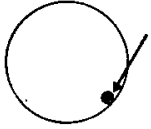

f

B

FIG. I A. - Technique d'autogreffe lorsque le lit porte-greffe est de dimensions très supérieures au greffon I-2 Taille du lit porte-greffe par excision d'un greffon de même diamètre

3-4 Le greffon précédent est découpé en lambeaux qui seront remis en place pour garnir le lit porte-greffe initial à l'exception d'une faible surface en position marginale.

4-5 Cette faible surface reçoit le greffon noir prélevé sur un autre site.

Frg. I B. - Représentation d l'échelle $1 / 2$ des lits porte-greffes, utilisés pour l'étude de l'influence de la surface du lit porte-greffe sur l'évolution du greffon

La surface repérée par une flèche représente le greffon noir en place.

Après anesthésie, l'animal dont les flancs ont été tondus, subit une incision cutanée antéro. postérieure dorsale. Les nerfs précédemment décrits sont dégagés pour pouvoir être chargés sur les électrodes de l'appareil. L'exploration des dermatomes correspondants est réalisée par déplacement d'une pointe de verre au contact de la peau. L'excitation nerveuse est mise en évidence grâce au courant recueilli sur l'écran. Le dermatome ainsi repéré, on ne se sert plus que de l'émission des hautparleurs pour en déterminer les limites.

I. Les dermatomes des branches dorsales des $9^{e}$ aux ${ }^{2} 3^{e}$ paires thoraciques ont un contour piriforme ; le sommet est situé au niveau de la colonne vertébrale, la partie élargie étant vers le bas. Une droite reliant l'angle postérieur du scapulum à la pointe du coxal passerait approximativement par leur milieu. Vers le bas, ces dermatomes se chevauchent largement. Chaque dermatome recouvre celui qui le précède et celui qui le suit d'environ la moitié de sa largeur.

2. Les dermatomes des branches perforantes des $9^{e}$ aux $13^{e}$ paires thoraciques ont un contour grossièrement elliptique. Leur partie supérieure chevauche les dermatomes des branches dorsales correspondantes. Vers le bas, les dermatomes des branches perforantes s'étendent jusqu'à la région médiane de l'abdomen. Pour ces branches perforantes, on trouve également un chevauchement de chaque dermatome par celui qui le précède et celui qui le suit.

3. Pour les paires lombaires étudiées ( $\mathrm{I}^{\mathrm{re}}$ et ${ }_{2} \mathrm{e}$ ), la forme des dermatomes est plus arrondie, les dermatomes des branches dorsales recouvrant la région du flanc située en avant du coxal. Les dermatomes des rameaux perforants sont plus ventraux.

Cette étude est illustrée par la figure 2. 
c) Technique d'énervation; contrôle postopératoire.

Il ressort de ce qui précède qu'une énervation efficace d'une large zone du flanc située en arrière du scapulum, peut être obtenue par section des branches dorsales et rameaux perforants des 5 dernières paires thoraciques et 2 premières paires lombaires ; c'est la méthode que nous avons employée. L'énervation est pratiquée immédiatement après la greffe au cours de la même anesthésie.

$\mathrm{La}$ vascularisation est soigneusement respectée.

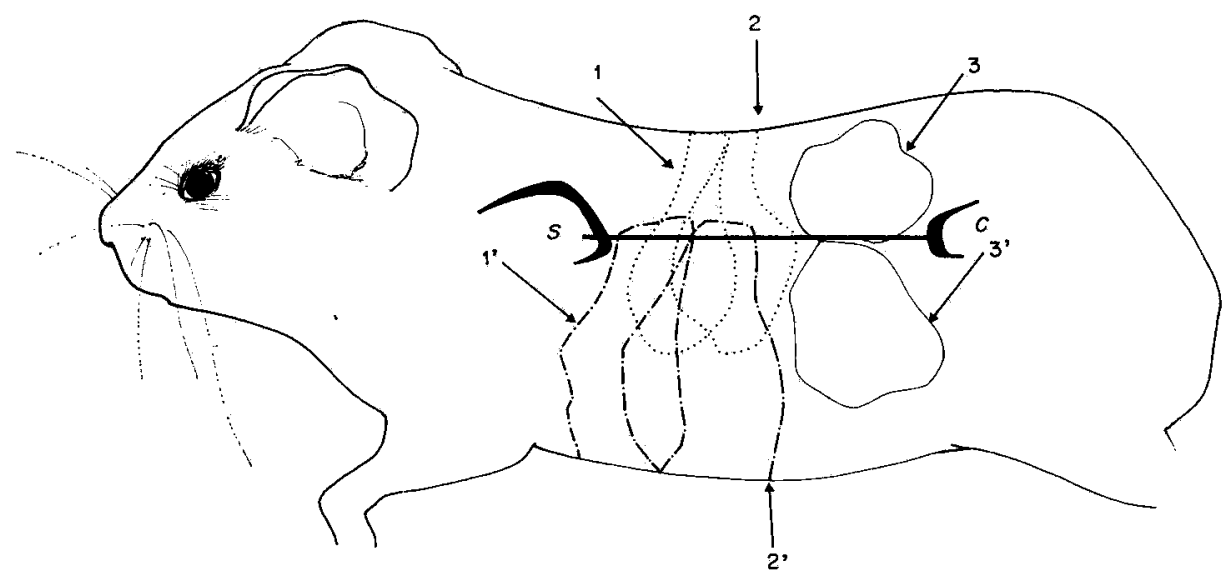

Fig. 2. - Les dermatomes du flanc chez le Cobaye

Nous n'avons représenté que les dermatomes de 2 paires thoraciques successives, et d'une paire lombaire $\mathrm{S}$ angle postérieur du scapulum,

$\mathrm{C}$ pointe du coxal

I et $\mathrm{I}^{\prime}$ dermatomes de la branche dorsale ( $\mathrm{I}$ ) et du rameau perforant $\left(\mathrm{I}^{\prime}\right)$ d'un nerf rachidien thoracique, 2 et $2^{\prime}$ même nomenclature pour le nerf rachidien suivant,

3 et $3^{\prime}$ même nomenclature pour un nerf lombaire.

\section{TABLEAU I}

Evolution de l'insensibilité du flanc énervé par section de nerfs, au cours des mois suivant l'opération

\begin{tabular}{c|c|c}
\hline $\begin{array}{c}\text { Nombre de mois écoulés } \\
\text { après la cicatrisation }\end{array}$ & $\begin{array}{c}\text { Nombre d'animaux } \\
\text { testés }\end{array}$ & $\begin{array}{c}\text { Nombre d'animaux } \\
\text { insensibles pour toute } \\
\text { la région du greffon }\end{array}$ \\
\hline 1 mois & 12 & 12 \\
\hline 2 mois & 12 & 12 \\
\hline 3 mois & 12 & 10 \\
\hline 4 mois & 12 & 9 \\
\hline 5 mois & 12 & 7 \\
\hline \hline
\end{tabular}

Nous avons également employé à titre de comparaison, et sur d'autres animaux, la méthode décrite par Fabian. Immédiatement après la greffe, et au cours de la même anesthésie, une incision cutanée semi-circulaire est pratiquée à l'avant du greffon dans la région duquel on veut obtenir 
l'énervation. Une deuxième incision symétrique vient compléter la première, après la cicatrisation de cette dernière. On forme ainsi une incision circulaire complète de $4 \mathrm{~cm}$ de diamètre, réalisée en 2 temps autour du greffon.

Pour toute énervation cutanée réalisée, on vérifie de façon hebdomadaire l'insensibilité à la piqûre pendant une durée de 5 mois. Ces explorations répétées montrent qu'il existe, comme prévu, une large zone insensible après l'opération, mais cette zone se rétrécit progressivement au cours des mois suivants. Il y a donc une restauration de la sensibilité cutanée. De ce fait, il est possible pour certains animaux, qu'en fin d'expérience, le greffon se retrouve en dehors de la zone insensible. C'est ce que nous avons consigné dans le tableau I pour les animaux énervés par section de nerfs.

On peut remarquer que l'insensibilité est pratiquement totale pendant les 3 premiers mois; elle persiste jusqu'à la fin des mesures pour plus de la moitié des animaux. $3^{\mathrm{e}}$ mois.

Pour les animaux énervés par incision circulaire, l'insensibilité ne subsiste guère au-delà du

\section{RÉSULTATS}

\section{A. - Étude de l'infuence de l'innervation superficielle sur l'évolution de la surface pigmentée}

Notre expérience a été conduite à partir d'un lot homogène de cobayes tricolores à poils ras, tous adultes et pesant initialement de 500 à 700 grammes. Nous n'avons retenu que les résultats correspondant aux animaux survivants à la fin de l'expérience, 5 mois après la greffe. La mortalité globale fut de I2 p. IOo. Dans ces conditions les résultats correspondent :

- pour l'énervation par section de nerfs à un lot de I 2 animaux, 7 mâles et 5 femelles;

— pour l'énervation par incision circulaire à un lot de to animaux, 5 mâles et 5 femelles.

Dans tous les cas, chaque animal reçoit 2 greffes pigmentées de $100 \mathrm{~mm}^{2}$ en territoire blanc : l'une est réalisée sur le flanc qui subit l'énervation, l'autre, sur le flanc intact, sert de témoin.

Chaque animal est donc son propre témoin.

Nous avons utilisé comme expression quantitative du phénomène, la racine carrée de la surface pigmentée exprimée en $\mathrm{mm}^{2}$, correspondant au greffon. Les variations de cette longueur peuvent être considérées comme proportionnelles à celle des dimensions de la tache pigmentée.

Comme nous l'avons montré, l'expression graphique des variations de la racine carrée de la surface fournit une courbe plus facilement interprétable que celle des variations de la surface elle-même (VouLot, I965).

Si l'on exprime le phénomène par la racine carrée de la surface en fonction du temps écoulé depuis la greffe, on voit que l'extension pigmentaire se fait d'abord à vitesse constante, puis ralentit au bout d'un temps déterminé pour se poursuivre à vitesse très faible (fig. 3 ).

Nous avons retenu pour cette étude, l'hypothèse d'un modèle linéaire, soit 2 segments de droite consécutifs, le second étant sensiblement horizontal.

Les valeurs moyennes successives des racines carrées des surfaces pour le flanc énervé, sont comparées aux moyennes correspondantes du flanc témoin, par la méthode des couples (test $t$ pour échantillons appariés). 


\section{TABLEAU 2}

Énervation par section de nerfs; comparaison des valeurs moyennes de la racine carrée de la surface mesurée correspondant au greffon, pour la série expérimentale (énervée) et la série témoin

La colonne I indique le moment auquel ont été faites les mesures, moment exprimé en nombre de semaines après la cicatrisation.

La colonne 2 précise s'il s'agit de greffes témoins, sans énervation (T) ou de greffes expérimentales en territoire énervé (E).

La colonne 3 donne la moyenne des surfaces pigmentées pour les séries $\mathrm{T}$ et $\mathrm{E}$.

La colonne 4, la moyenne des racines carrées des surfaces pigmentées pour les séries $T$ et $E$.

La colonne 5 , la valeur de $t$ correspondant à la comparaison des moyennes expérimentales (E) et témoin ( $\mathrm{T})$ de la colonne 4 .

Pour le nombre de degré de liberté $(v=\mathrm{II})$ les différences caractérisées par une valeur de $t$ (Student) supérieure ou égale à 2,20 doivent être considérées comme significatives à 0,05 près.

\begin{tabular}{|c|c|c|c|c|c|c|c|c|c|}
\hline 1 & 2 & 3 & 4 & 5 & 1 & 2 & 3 & 4 & 5 \\
\hline Semaines & & $1 / n \sum_{s}$ & $1 / n \sum \sqrt{s}$ & $t$ & Semaines & & $1 / n \sum s$ & $1 / n \sum \sqrt{s}$ & \\
\hline 1 & $\begin{array}{l}\mathrm{T} \\
\mathrm{E}\end{array}$ & $\begin{array}{l}43 \\
53\end{array}$ & $\begin{array}{l}6,36 \\
7,06\end{array}$ & 1,30 & 10 & $\begin{array}{l}\mathrm{T} \\
\mathbf{E}\end{array}$ & $\begin{array}{l}144 \\
203\end{array}$ & $\begin{array}{l}11,87 \\
14,02\end{array}$ & 3,31 \\
\hline 2 & $\begin{array}{l}\mathrm{T} \\
\mathrm{E}\end{array}$ & $\begin{array}{l}48 \\
6^{\prime} t\end{array}$ & $\begin{array}{l}6,68 \\
7,79\end{array}$ & 1,26 & 11 & $\begin{array}{l}\mathrm{T} \\
\mathrm{E}\end{array}$ & $\begin{array}{l}158 \\
236\end{array}$ & $\begin{array}{l}12,56 \\
15,16\end{array}$ & $3,9^{\prime} t$ \\
\hline 3 & $\begin{array}{l}\mathrm{T} \\
\mathrm{E}\end{array}$ & $\begin{array}{l}59 \\
83\end{array}$ & $\begin{array}{l}7,45 \\
8,93\end{array}$ & 2,09 & 12 & $\begin{array}{l}\mathrm{T} \\
\mathrm{E}\end{array}$ & $\begin{array}{l}172 \\
235\end{array}$ & $\begin{array}{l}12,87 \\
15,08\end{array}$ & 2,93 \\
\hline 4 & $\begin{array}{l}\mathrm{T} \\
\mathrm{E}\end{array}$ & $\begin{array}{r}71 \\
105\end{array}$ & $\begin{array}{r}8,28 \\
10,07\end{array}$ & 2,57 & 13 & $\begin{array}{l}\mathrm{T} \\
\mathrm{E}\end{array}$ & $\begin{array}{l}177 \\
254\end{array}$ & $\begin{array}{l}13,08 \\
15,74\end{array}$ & 4,76 \\
\hline 5 & $\begin{array}{l}\mathrm{T} \\
\mathrm{E}\end{array}$ & $\begin{array}{r}82 \\
108\end{array}$ & $\begin{array}{r}8,88 \\
10,19\end{array}$ & 2,02 & 14 & $\begin{array}{l}\mathrm{T} \\
\mathrm{E}\end{array}$ & $\begin{array}{l}175 \\
265\end{array}$ & $\begin{array}{l}13,08 \\
16,23\end{array}$ & 4,41 \\
\hline 6 & $\begin{array}{l}\mathrm{T} \\
\mathrm{E}\end{array}$ & $\begin{array}{r}88 \\
112\end{array}$ & $\begin{array}{r}9,20 \\
10,34\end{array}$ & 2,30 & 15 & $\begin{array}{l}\mathrm{T} \\
\mathrm{E}\end{array}$ & $\begin{array}{l}189 \\
272\end{array}$ & $\begin{array}{l}13,40 \\
16,18\end{array}$ & 4,37 \\
\hline 7 & $\begin{array}{l}\mathrm{T} \\
\mathrm{E}\end{array}$ & $\begin{array}{l}106 \\
142\end{array}$ & $\begin{array}{l}10,05 \\
11,76\end{array}$ & 2,32 & 19 & $\begin{array}{l}\mathrm{T} \\
\mathrm{E}\end{array}$ & $\begin{array}{l}194 \\
315\end{array}$ & $\begin{array}{l}13,52 \\
17,29\end{array}$ & 6,15 \\
\hline 8 & $\begin{array}{l}\mathrm{T} \\
\mathrm{E}\end{array}$ & $\begin{array}{l}112 \\
158\end{array}$ & $\begin{array}{l}10,41 \\
12,40\end{array}$ & 3,10 & 21 & $\begin{array}{l}\mathrm{T} \\
\mathbf{E}\end{array}$ & $\begin{array}{l}219 \\
339\end{array}$ & $\begin{array}{l}14,41 \\
18,01\end{array}$ & 5,33 \\
\hline 9 & $\begin{array}{l}\mathrm{T} \\
\mathrm{E}\end{array}$ & $\begin{array}{l}127 \\
184\end{array}$ & $\begin{array}{l}11,14 \\
13,37\end{array}$ & 3,30 & & & & & \\
\hline
\end{tabular}

- Dans le cas de 1'expérimentation réalisée par section de nerfs, les résultats sont consignés dans le tableau 2. On voit que la différence entre la moyenne expérimentale et la moyenne témoin devient continuellement significative, pour le seuil de probabilité considéré, au bout de 5 semaines. L,es différences sont particulièrement nettes à partir du $3^{\mathrm{e}}$ mois.

- Pour l'expérimentation par incisions circulaires, nous n'avons pas rapporté le détail des résultats. Ces derniers sont comparables aux précédents, mais les diffé- 
rences sont moins marquées. L'application du test $t$, toutefois montre que ces différences sont significatives dès la $6^{\mathbf{e}}$ semaine et le restent jusqu'à la fin de l'expérience.

La figure 3 illustre graphiquement les résultats obtenus par section de nerf. La courbe expérimentale présente une allure comparable à la courbe témoin mais les pentes sont modifiées $\left({ }^{\mathbf{1}}\right)$.

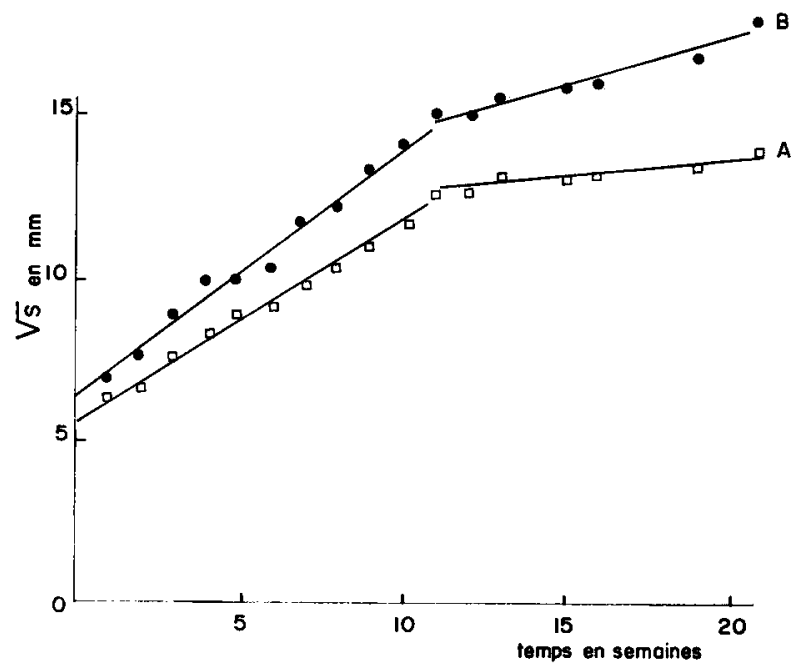

FIG. 3. - Expérience d'énervation par section de nerfs. - Évolution du greffon en fonction du temps

En abscisse, le temps en nombre de semaines écoulées depuis la cicatrisation postopératoire.

En ordonnée, la valeur moyenne de la racine carrée de la surface mesurée.

La courbe A correspond aux greffes implantées sur le fianc témoin non énervé.

La courbe B correspond aux greffes implantées sur le flanc expérimental ayant subi l'énervation par section de nerf.

La comparaison des pentes correspondant au deuxième alignement pour les courbes témoin et expérimentale montre que ces dernières sont significativement différentes $(0,0909$ et 0,2864$)$. Dans le cas de la courbe expérimentale, le ralentissement de la vitesse d'extension pigmentaire représenté par le deuxième segment de droite est donc moins important que dans le cas de la courbe témoin.

On peut retrouver d'ailleurs ces conclusions en appliquant aux deux courbes témoin et expérimentale, considérées chacune dans leur ensemble, un test de linéarité. Pour la courbe témoin nous sommes amenés à conclure à une impossibilité de 1'hypothèse linéaire. Le très net ralentissement de la vitesse d'extension pigmentaire dans la deuxième partie de cette courbe explique ce résultat. Pour la courbe expérimentale au contraire, une valeur de $\mathrm{F}$ calculée, inférieure à l'unité, n'exclut pas l'éventualité d'une droite unique, ce qui impliquerait la persistance d'une vitesse d'extension pigmentaire continue tout au long de l'expérience.

( $\left.{ }^{(}\right)$La pente pour le premier alignement de la courbe témoin semble très différente de la valeur fournie dans une publication précédente (VoULOT, I965) soit $b=0,6199$ au lieu de $b=0.1$ I3I. En fait, le temps était dans le deuxième cas exprimé en jours, alors qu'il l'est ici en semaines. Un calcul élémentaire montre que nous avons bien un même ordre de grandeur. 


\section{B. - Étude de l'influence de la surface du lit porte-greffe sur l'évolution du greffon pigmenté}

Il est évident que l'excision d'un lit porte-greffe provoque une destruction locale de l'innervation superficielle.

L'existence d'un facteur nerveux dans le déroulement de l'extension pigmentaire implique donc une influence des dimensions du lit porte-greffe. C'est une telle relation que nous avons cherché à vérifier.

Six surfaces différentes de lit porte-greffe, furent utilisées, allant de $10 \mathrm{~mm}^{2}$ à $500 \mathrm{~mm}^{2}$ qui est l'ordre de grandeur maximum que l'on puisse envisager pour un cobaye adulte (fig. I, B).

\section{TABLEAU 3}

Influence de la taille du lit porte-greffe sur les limites dé l'extension pigmentaire

Pour chaque date de mesure considérée ( $\mathrm{I}, 3,4,5$ et 6 mois après la cicatrisation) on effectue une analyse de la variance à $I$ facteur contrôlé (taille du lit porte-greffe).

Les résultats sont consignés ci-dessus. La valeur de $\mathrm{F}$ de Snedecor correspond au seuil 0,05 .

Le nombre de degrés de liberté montre que le total des mesures augmente après la Ire semaine. Cela est dû au fait qu'en raison d'une cicatrisation incomplète, certaines mesures impossibles au début l'ont été par la suite.

\begin{tabular}{|c|c|c|c|c|c|c|}
\hline $\begin{array}{l}\text { Date des } \\
\text { mesures }\end{array}$ & $\begin{array}{l}\text { Sources de } \\
\text { variations }\end{array}$ & $\begin{array}{c}\text { Somme } \\
\text { des carrés } \\
\text { des écarts }\end{array}$ & $\begin{array}{l}\text { Nombre } \\
\text { de degrés } \\
\text { de liberté }\end{array}$ & $\begin{array}{l}\text { Carrés } \\
\text { moyens }\end{array}$ & F calculé & $\mathrm{F}$ théorique \\
\hline $1^{\text {er }}$ mois & $\begin{array}{l}\text { traitement } \\
\text { résiduelle } \\
\text { totale }\end{array}$ & $\begin{array}{r}392 \\
6915 \\
7307\end{array}$ & $\begin{array}{r}5 \\
54 \\
59\end{array}$ & $\begin{array}{r}78,40 \\
128,06\end{array}$ & 0,61 & 2,53 \\
\hline $3^{e}$ mois & $\begin{array}{l}\text { traitement } \\
\text { résiduelle } \\
\text { totale }\end{array}$ & $\begin{array}{l}11046 \\
54272 \\
65318\end{array}$ & $\begin{array}{r}5 \\
61 \\
66\end{array}$ & $\begin{array}{r}209,20 \\
889,70\end{array}$ & $2,4.8$ & 2,37 \\
\hline $4^{\theta}$ mois & $\begin{array}{l}\text { traitement } \\
\text { résiduelle } \\
\text { totale }\end{array}$ & $\begin{array}{l}16037 \\
429+2 \\
58979\end{array}$ & $\begin{array}{c}5 \\
46 \\
51\end{array}$ & $\begin{array}{r}3207,40 \\
933,52\end{array}$ & 3,43 & 2,53 \\
\hline $5^{\mathrm{e}}$ mois & $\begin{array}{l}\text { traitemenit } \\
\text { résiduelle } \\
\text { totale }\end{array}$ & $\begin{array}{r}39378 \\
67169 \\
106547\end{array}$ & $\begin{array}{r}5 \\
44 \\
49\end{array}$ & $\begin{array}{l}7875,60 \\
1526,56\end{array}$ & 5,16 & 2,53 \\
\hline $6^{e}$ mois & $\begin{array}{l}\text { traitement } \\
\text { résiduelle } \\
\text { totale }\end{array}$ & $\begin{array}{r}30676 \\
86823 \\
117499\end{array}$ & $\begin{array}{r}5 \\
36 \\
41\end{array}$ & $\begin{array}{l}6135,20 \\
2411,75\end{array}$ & 2,54 & 2,53 \\
\hline
\end{tabular}

Le greffon noir a dans tous les cas Io $\mathrm{mm}^{2}$. Notons $a$, le lit porte-greffe de Io $\mathrm{mm}^{2}$, $b$, celui de $25 \mathrm{~mm}^{2}, c$, celui de $50 \mathrm{~mm}^{2}, d$, celui de $150 \mathrm{~mm}^{2}, e$, celui de $300 \mathrm{~mm}^{2}, f$, celui de $500 \mathrm{~mm}^{2}$.

Comme dans l'expérience précédente, où chaque animal était son propre témoin, nous avons cherché à éliminer au maximum les variations individuelles. Chaque 
cobaye porte donc 2 greffes de type différent, et les types de greffes doivent être combinées 2 à 2 de toutes les manières possibles. Le nombre minimum d'animaux correspond alors au nombre de combinaisons des 6 types de greffes prises 2 à 2, soit 15. Mais pour avoir un nombre suffisant de répétitions, nous avons réalisé 3 fois chaque combinaison, soit $3 a b, 3 a c, 3 a d, 3 a e, 3 a f, 3 b c, 3 b d, 3 b e, 3 b f, 3 c d, 3 c e$, $3 c f, 3 d e, 3 d f, 3 e f$. Ce qui correspond à 35 animaux en expérience.

Les mesures des taches pigmentées ont été effectuées, $x, 3,4$ et 5 mois après la cicatrisation des greffes.

Le problème étant de savoir s'il existe une relation entre la surface du lit portegreffe et celle atteinte par la tache pigmentée au moment de la mesure, une analyse de la variance à un facteur contrôlé s'impose. L,e facteur contrôlé est la taille variant de $a$ à $f$ du lit porte-greffe.

Le détail de ces analyses de variance est consigné dans le tableau 3.

La valeur de $F$ calculée est comparée à celle du $F$ de Snedecor (probabilité de 0,05$)$. La variance due au traitement devient significativement supérieure à la variance résiduelle, 3 mois après la greffe, elle le reste jusqu'à la fin de l'expérience, en marquant toutefois un net maximum au $5^{\mathbf{e}}$ mois.

Nous devons donc conclure à une influence déterminante des dimensions de la surface excisée lors de la réalisation du lit porte-greffe sur l'importance ultérieure de l'extension pigmentaire.

Signalons que nous avons montré dans une expérimentation préalable que la taille du greffon, au contraire, ne semble pas intervenir. En effet, après avoir implanté dans les mêmes conditions des microgreffes de peau noire et des greffons pigmentés de $\mathrm{I} \mathrm{cm}^{2}$, nous avons vu la différence entre les surfaces pigmentées des deux séries diminuer progressivement après la greffe, pour finalement devenir non significative dès la $6^{\mathrm{e}}$ semaine.

\section{DISCUSSION}

Nous venons de montrer que dans les zones cutanées où l'on détruit l'innervation, l'extension pigmentaire est plus étendue qu'ailleurs, ce qui, en d'autres termes, signifie que l'innervation en place doit inhiber l'extension pigmentaire.

Il est certes prématuré de vouloir expliquer le mécanisme de l'action du système nerveux périphérique, mais l'on peut fort bien, par contre, interpréter l'ensemble des faits expérimentaux à la lumière de cette hypothèse d'inhibition.

\section{Analyse des faits expérimentaux}

Dans le cas le plus ordinaire, celui des greffes témoins sur le flanc intact par exemple, l'extension pigmentaire se produit dans la zone énervée consécutive à l'excision du lit porte-greffe. Cette zone est plus grande que la tache pigmentée mesurée à l'origine car le greffon ne prend que sur une partie du lit porte-greffe. Dès que la zone pigmentée en cours d'extension dépasse les limites de la zone énervée, l'innervation en place freine progressivement le phénomène. Si l'on considère la figure 3 , nous voyons que pour la courbe A, le palier sub-horizontal (phase d'inhibition de l'extension pigmentaire) s'établit 3 mois environ après la greffe. A ce moment, la 
grandeur représentée graphiquement (moyenne des racines carrées des surfaces mesurées) est peu différente de $\mathrm{I} 2 \mathrm{~mm}$. Si ces surfaces mesurées sont considérées comme circulaires, le rayon moyen correspondant serait de $6,7 \mathrm{~mm}$. Or, le rayon d'un cercle de $100 \mathrm{~mm}^{2}$ de surface (lit porte-greffe initial et par conséquent zone énervée correspondante) est de $5,6 \mathrm{~mm}$. Nous voyons donc que l'effet d'inhibition est devenu sensible, lorsque le rayon correspondant à la surface pigmentée dépasse légèrement ( $\mathrm{mm}$ ) celui de la zone énervée.

Dans le cas d'énervation chirurgicale, l'extension pigmentaire ne devrait s'arrêter que bien plus tard. En fait, nous voyons (fig. 3 ) que sur la courbe B correspondante, la vitesse de l'extension pigmentaire diminue après le $3^{\mathrm{e}}$ mois. Toutefois cette diminution est beaucoup moins rapide que dans le cas du témoin. Nous avons précédemment signalé que les zones insensibles (énervées) voient leurs surfaces diminuer en cours d'expérience. Cette restauration nerveuse explique sans doute, dans le cas de la courbe $B$, la diminution observée de la vitesse initiale d'extension pigmentaire. Notons cependant que l'on peut également considérer cette diminution graphiquement décelable, comme due aux aléas d'échantillonnage : en effet, le test de linéarité pratiqué sur l'ensemble de la courbe $B$ ne permet pas de conclure au rejet d'un modèle linéaire, à la différence de celui pratiqué sur la courbe $\mathrm{A}$.

En conclusion, si notre hypothèse d'une action inhibitrice de l'innervation cutanée sur l'extension pigmentaire peut être retenue, il faut remarquer néanmoins qu'elle nous permet essentiellement d'expliquer le ralentissement et les limites du phénomène considéré, et non pas à rendre compte de son déclenchement à la suite de la greffe. Nous ne pouvons, en effet, affirmer que la seule disparition de l'innervation cutanée détermine la migration des mélanocytes. Une analyse expérimentale plus poussée s'impose.

\section{Quelques remarques sur les rapports entre le système nerveux et la physiologie des mélanocytes}

Nos observations doivent être situées dans le cadre plus général des influences du système nerveux sur la physiologie des cellules pigmentaires.

On a décrit au niveau de certains groupes d'Invertébrés une action du système nerveux sur la pigmentation (mélanique ou non). Bien plus, dans certains cas, l'expérimentateur est conduit à des observations qui rappellent curieusement les nôtres. C'est le cas par exemple pour le Lombricien Eisenia foetida. Chez cet animal, LASALLE (I966) observe au niveau de plaies énervées, et dans les I5 jours qui suivent l'opération, une augmentation de la pigmentation. VAL_EMBOIs (I967) sur la même espèce, note un état d'activité intense des cellules pigmentaires dans les hétérogreffes de paroi en dégénérescence. Cet auteur conclut à l'existence d'un contrôle nerveux de la pigmentation, inhibé lors de la greffe, le greffon étant, dans ce cas, difficilement pénétré par les éléments nerveux du porte-greffe.

Chez les Vertébrés inférieurs on a décrit depuis longtemps une action de l'innervation superficielle sur les variations de la pigmentation cutanée. Nous remarquons cependant que les travaux récents dans ce domaine, ne confirment pas les résultats initiaux (SNELL-Kulovitch, I967). Par contre, on connait bien l'action de divers agents chimiques sur les variations pigmentaires de la peau des Batraciens (LERNER, 
I960). Nous rappellerons entre autres, l'action mélanisante d'hormones hypophysaires ( $\alpha$ et $\beta$ MSH, ACTH) et, à l'inverse, l'activité " éclaircissante " de la mélatonine, adrénaline, noradrénaline, acétyl-choline ..., etc. Il est frappant de reconnaître parmi ces derniers corps les médiateurs chimiques du système nerveux. Leur action est immédiate, non seulement sur les mélanophores in situ, mais également sur des cellules pigmentaires en culture de tissus dans le cas de la larve de Salamandre (Noval,ES et NOVALES, I966).

Enfin la mélatonine, qui constitue l'agent éclaircissant le plus actif sur la peau de Batracien, a été mise en évidence par LERNER au niveau des nerfs périphériques des Mammifères.

Si de tels recoupements paraissent prometteurs, il faut cependant ne pas ignorer les différences fondamentales qui existent entre la physiologie du mélanophore des Batraciens et celle du mélanocyte des Mammifères :

- les effets physiologiques des agents chimiques cités plus haut sur les mélanophores concernent essentiellement des mouvements de particules mélaniques à l'intérieur des cellules pigmentaires, et en aucun cas les migrations de ces cellules;

- à la différence des hormones de mélanisation, les substances " éclaircissantes " citées plus haut ne sont pas actives sur les mélanocytes de Mammifère.

Un important travail expérimental reste à faire pour justifier chez le Mammifère les rapprochements que nous venons de suggérer.

Reçu pour publication en mars 1968.

\section{SUMMARY}

\section{EFFECT OF LOCAL DENERVATION ON PIGMENT SPREAD}

IN THE GUINEA PIG

The effect of local denervation in mammalian (Guinea pig) pigment spread has been investigated.

Denervation is performed by superficial nerve sections. Before denervation an electrophysiologic method has been used to locate dermatomas on the guinea pig's chest. After denervation the insensibility of denervated areas has been tested weekly till the end of the experiment.

A quantitative graft-area measurement investigation, shows that pigment spread is larger after denervation than on innervated skin. The decrease of pigment spread speed is more important on intact skin than on denervated.

This suggests that nervous system inhibits pigment spread.

Skin graft bed cutting induces a local denervation. We found that when skin graft bed is increased, an enlargement of the correspondant pigment spread area occurs.

\section{REMERCIEMENTS}

Je tiens à remercier mes collègues du Laboratoire de Physiologie de l'Institut national des Sciences appliquées de Lyon qui ont mis à ma disposition le matériel nécessaire à la partie électrophysiologique de ce travail. 


\section{RÉFÉRENCES BIBLIOGRAPHIQUES}

Billingham R. E., Medawar P. B., 1948. Pigment spread and cell heredity in guinea pigs'skin. Heredity, 2, 29-47.

Biluingham R. E., Silvers W. K., 1964. The current status of the pigment spread problem. $J$. Invest. Dermatol., 42, 9-14.

Fabian G. Y., I953. Spread of black pigment on the denervated skin of guinea pigs. Acla biol. Acad.Sci. Hung., 4, 471-79.

LASALLE A. M., 1966. Róle du système nerveux dans la cicatrisation des plaies de la paroi du corps chez le Lombricien Eisenia foetida SAv. Thèse de $3^{\mathrm{e}}$ cycle, Bordeaux.

LERNER A. B., r960. Hormonal control of pigmentation. Ann. Rev. Med., 11, 187-r94.

LERNER A. B., 1960. Melatonin Intersociety symposium on new and neglected hormones. Fed. Proc., 19, 590-592.

Novales R. R., Novales J. B., 1966. Cytological and ultrastructural aspects of Amphibian Melanophore Control. In Structure and Control of the melanocyte, ed. by G. DELLA PORVA and O. MUnLBOCK, Springer Verlag-Berlin, Heidelberg New-York, 52-59.

SNELL R. S., KULOVICTH S., I 967 . Nerve stimulation and the movement of melanin granule in the pigment cell of the Frog's web. Invest. Derm., 18, 438-443.

VALEmbors P., 1967. Infrastructure des cellules pigmentaires des Lombriciens et étude de leur rôle dans les hétérogreffes de paroi du corps. C. R. Acad. Sci., Paris, 265, 362-364.

Voulot C., I965. Étude quantitative du phénomène d'extension pigmentaire chez le Cobaye. C. R. Acad. Sci. Paris, 261, 4517-4520. 